\title{
Handgrip Strength Features in Rheumatoid Arthritis Patients Assessed Using an Innovative Cylindrical-Shaped Device: Relationships With Demographic, Anthropometric and Clinical Variables
}

\author{
Fausto Salaffi ${ }^{1}$ (D) Marina Carotti ${ }^{2}$ (D) . Sonia Farah $^{1}$ (D) $\cdot$ Luca Ceccarelli $^{3}$ (D) Marco Di Carlo $^{1}$ (D)
}

Received: 6 September 2021 / Accepted: 4 October 2021 / Published online: 9 October 2021

(c) The Author(s) 2021

\begin{abstract}
To investigate the relationship between handgrip strength (HGs) features, evaluated with an innovative cylindricalshaped grip device, and demographic, anthropometric and clinical variables, in patients with rheumatoid arthritis (RA). Consecutive RA patients were prospectively enrolled for this cross-sectional study. For each patient were collected demographic, anthropometric, clinical data related to disease activity. HGs was assessed in terms of area under the forcetime curve (AUC-FeT), peak grip force and time to reach the curve plateau. The correlations between the variables were studied with the Spearman's rho correlation coefficient. The receiver operating characteristic (ROC) curve analysis was used to test the discriminant accuracy of HGs features in identifying patients in moderate/high disease activity. A multivariate analysis was performed to estimate the contribution of covariates on the AUC-FeT. A significant correlation was found among AUC-FeT, age, Simplified Disease Activity Index (SDAI), Ultrasound-Clinical Arthritis Activity (US-CLARA) (all at $p<0.0001)$, and body mass index (BMI) $(p=0.0001)$. Any correlation was found between HGs and radiographic damage. The discriminatory power of AUC-FeT was good [area under-ROC curve $=0.810$ (95\% CI 0.746-0.864)]. Variables significantly associated with AUC-FeT in multivariate analysis were age $(p=0.0006)$, BMI $(p=0.012)$, gender $(p=0.004)$, SDAI $(p=0.047)$ and US-CLARA $(p=0.023)$. HGs is negatively influenced by demographic (gender and age), anthropometric (BMI), and disease activity variables (SDAI and US-CLARA). These findings highlight the role of HGs in RA patients' functional impairment and disability.
\end{abstract}

Keywords Rheumatoid arthritis · Handgrip strength · Cylindrical-shaped dynamometer · Disease activity indices · Disability

\section{Abbreviations \\ HDA High disease activity \\ MDA Moderate disease activity}

\section{Key points}

- Traditional dynamometers evaluate only the isometric unidirectional force.

- The innovative cylindrical-shaped dynamometer allows to evaluate the area under the force-time curve, peak grip force and time to reach the curve plateau.

- Handgrip strength in patients with rheumatoid arthritis is negatively influenced by both demographic (gender and age), anthropometric (BMI), and disease activity variables (SDAI and US-CLARA).

This article is part of the Topical Collection on Clinical Systems

Marco Di Carlo

dica.marco@yahoo.it

Extended author information available on the last page of the article

\section{LDA Low disease activity}

REM Remission

\section{Introduction}

Rheumatoid arthritis (RA) is a chronic inflammatory disease that affects people all over the world, with a $0.5 \%$ of prevalence in Italy [1]. RA affects a number of joints, with a large percentage of patients experiencing symptoms in hands and wrists. Joint pain and swelling are common symptoms, resulting in diminished hand strength and movement impairment [2]. Although there is some evidence that measures of disease activity may not fully reflect the regional impact of RA on the hands, indices such as the disease activity score with 28 joint count (DAS28) are the cornerstore of the assessment RA patients [3]. It might be argued that evaluating hand 
function should be done separately from a RA patient's disease activity evaluation [4]. Handgrip strength (HGs) has been shown to correlate with both objective and patient-reported outcome measures (PROMs) of disease activity and function [5, 6]. HGs can be used to assess a patient's ability to return to routine activities and job, as well as to track progress and compare the efficacy of different treatment strategies. Results coming from the VErA (Very Early Rheumatoid Arthritis) project has revealed that lower HGs in RA patients are related with a larger economic burden [7].

Several studies support the use of ultrasound (US) to detect and monitor joint inflammation and bone deterioration in individuals with RA. Although the joint count remains the most specific marker for RA assessment, several studies have recently been published that support the use of US to detect and monitor joint inflammation and bone deterioration in individuals with RA [8]. Current European Leaugue Againts Rheumatims (EULAR) recommendations on the use of imaging modalities in RA recognize the high sensitivity of US to detect joint pathologies, suggesting its extensive use in the assessment of disease activity [9]. Power Doppler US (PDUS) has enhanced its sensitivity in identifying low velocity flow at the microvascular level, making it a helpful method for assessing the degree of inflammation and disease activity in RA [10, $11]$.

Multimodal disease activity indices combining clinical and ultrasonographic data seem to have better reliability than clinical assessment alone [12, 13]. An Italian study conducted on a cohort of RA patients starting treatment with abatacept, demonstrated good metrological properties (validity, responsiveness and interpretability) compared to traditional disease activity indices, of a new composite index called Ultrasound-Clinical Arthritis Activity (US-CLARA) [14]. To date, there are no studies that have compared HGs with multimodal disease activity indices of in RA.

At present, it is also unknown whether or not hands radiological damage is associated with a reduction in HGs. With currently available tools such as the Simple Erosion Narrowing Score (SENS) [15], a simplified instrument based on the Sharp-van der Heijde Score (SHS), the assessment of radiographic damage is also accessible in daily clinical practice.

The evaluation of HGs can be performed with different types of dynamometer. To the best of our knowledge, no study to date has assessed HGs in RA using a cylindricalshaped dynamometer.

Starting from these assumptions, the objective of this study was to investigate the relationship between HGs features and clinical variables, composite and multimodal disease activity indices, and radiographic damage.

\section{Methods}

\section{Design and study population}

In this observational cross-sectional study, consecutive RA patients were prospectively enrolled from January 2019 to December 2020. Patients were enrolled in the outpatients clinic of the Rheumatology Clinic of the Università Politecnica delle Marche, "Carlo Urbani" Hospital, Jesi (Ancona), Italy. The inclusion criteria were: age between 18 and 80 years, diagnosis of RA made according to the 2010 American College of Rheumatology (ACR)/EULAR criteria [16]. Exclusion criteria were represented by the presence of: limitations (such as visual impairments, lack of command of the Italian language) determining the impossibility to complete the clinimetric assessment, concurrent life-threatening disorders (such as active neoplasms, heart failure, severe chronic obstructive pulmonary disease, multiple sclerosis, extracorporeal dialysis), coexisting inflammatory crystal arthropaties (such as gout or calcium pyrophosphate deposition disease) of coexisting conditions capable of determining a reduction of HGs or altering the clinimetric assessment such as fibromyalgia.

\section{Assessment of demographic, anthropometric variables and comorbidities}

Age, sex, disease duration (defined as time since diagnosis), level of education (primary, secondary, and university), body mass index (BMI), concomitant medication with glucocorticoids and traditional conventional syntethic disease modifying anti-rheumatic drugs (csDMARDs) or biological DMARDs (bDMARDs), and comorbidities were all included in the study. The comorbidities burden was estimated using the modified Rheumatic Disease Comorbidity Index (mRDCI) [17, 18], computed with the formula: $1 *$ lung disease and [2* (myocardial infarction, other cardiovascular illnesses, or stroke) or $1 *$ hypertension] and $1^{*}$ (ulcer or other gastrointestinal disorders) and $2 *$ kidney disease and $1 *$ if BMI is $>30 \mathrm{~kg} / \mathrm{m}^{2}$ or $2 *$ if BMI is $>35 \mathrm{~kg} / \mathrm{m}^{2}$, and 1 for each of diabetes, fracture, depression, and cancer. The final mRDCI score ranges from 0 to 12 [17].

\section{Laboratory investigations}

Standard laboratory methods were used to assess the erythrocyte sedimentation rate (ESR) (normal values $15 \mathrm{~mm} / 1 \mathrm{st}$ hour in men and $20 \mathrm{~mm} / 1$ st hour in women), the C-reactive protein (CRP) (expressed in $\mathrm{mg} / \mathrm{dl}$ ), the IgM-rheumatoid 
factor (RF) (measured by nephelometric method, Image Beckman), and the anti-citrullinated protein antibodies (ACPA) (measured by ImmunoFluoroMetric Assay, EliA CCP, ImmunoCAP 250, Phadia S.r.l., Italy).

\section{Assessment of HGs}

HGs was measured using a cylindrical-shaped grip device with five force sensors (FRS-402, manufacted by Interlink Electronics) attached to a microcontroller (Arduino Mega 2560). This dynamometer represents a prototype and is not yet available on a large scale.

The grip force was measured by the grip device every $5 \mathrm{~s}$ for $30 \mathrm{~s}$. This assessment allowed to draw the force-time $(\mathrm{FeT})$ curves. The FeT curves are a graphical representation of muscular contraction force over time. The principal outcome for the purposes of this study was the estimation of the area under the FeT curve (AUC-FeT), with peak grip force and time to reach the curve plateau as secondary outcomes.

The HGs features were measured twice in the dominant with an interval of $5 \mathrm{~min}$ for fatigue recovery between the two measurements. The mean of the two values of the three parameters was used for the analyses [19]. HGs was assessed according to the American Society of Hand Therapists' recommendations for subject positioning [20]: patients in seated position, the shoulder adducted and neutrally rotated, the wrist was slightly extended, the elbow was flexed to roughly 90 degrees, and the forearm was in neutral position. Instructions were presented in a consistent manner to all patients.

\section{Disease activity indices}

The Simplified Disease Activity Index (SDAI) and the USCLARA were respectively used as composite and multimodal disease activity indices.

The SDAI is the algebraic sum of five unweighted, untransformed variables: 28 -joint swollen and tender joints counts (SJC and TJC, respectively), physician and patient disease activity assessment (PhGA and PaGA, respectively) on 11-point numerical rating scales (NRS), and CRP (in mg/ $\mathrm{dl}$ ). The SDAI range is $0-86$. Remission (REM), low disease activity (LDA), and moderate disease activity (MDA) has the predefined cut-off values of $3.3,11$ and 26 , respectively [21].

The US-CLARA is a composite index that combines the Recent Onset Arthritis Disability (ROAD) questionnaire, the self-administered TJC, and the US evaluation into a single measure of disease activity [14, 22, 23]. The US examinations were carried out with a MyLab Class C US system (Esaote S.p.A., Genoa, Italy), equipped with a 6-18 MHz linear probe. A full description of the US-CLARA is provided in the validation study [14]. The final score of US-CLARA ranges from 0 to 10 , and the following interpretability cut-off values have been proposed: REM $<2.0 ; 2.0 \leq \mathrm{LDA}<3.0$; $3 \leq \mathrm{MDA}<4.8$; and high disease activity (HDA) $>4.8$.

\section{Radiographic assessment}

Hands, wrists, and foot radiography were performed on all of the patients. The images were evaluated using the SENS by two experienced readers (FS and MC). The SENS scoring system evaluates the same joints as the SHS scoring technique. While SHS uses a $0-4$ semiquantitative scale for joint space narrowing (JSN) and a $0-5$ scale for erosions, SENS simply determines if an erosion is present (score 1) and whether JSN is present (score 1). The SENS has a maximum possible score of 86 [15].

\section{Statistical analysis}

The MedCalc Statistical Software, version 19.0 (Ostend, Belgium), for Windows XP was used to analyze the data. For categorical variables, general descriptive statistics were described using numbers and percentages, while continuous variables were described using mean with standard deviation (SD), and median with interquartile range (IQR). The normal distribution was tested with the Kolmogorov-Smirnov test. Where appropriate, the percent differences between the groups were examined using a chi-square or Fisher's exact test. The Mann-Whitney U test was used to compare continuous variables among categories of grouped data.

The Spearman's rho was used to measure the degree of correlation between the variables.

Patients were categorized for disease severity states according to SDAI. The AUC-FeT differences according the categories were tested with the one-way analysis of variance (ANOVA).

Then, a receiver operating characteristic (ROC) curve analysis was used to test the discriminant accuracy of HGs measurements using SDAI (patients were categorized in MDA + HDA vs REM + LDA) as external criterion. AUC-ROC values between 0.7 and 0.8 suggest a fair discrimination, whereas values above 0.8 suggest a good discrimination. We used non-parametric resampling and the bias-corrected and accelerated approach to construct 95\% confidence intervals (CIs) on 1000 bootstrapped samples. The non-parametric Wilcoxon signed ranks test was used to calculate and compare the AUC-ROCs.

Finally, the relative effect of individual variables (including among covariates age, sex, education, disease duration, BMI, mRDCI, SDAI, US-CLARA and SENS) on the AUCFeT (considered as dependent variable) was assessed using a multivariate regression model approach. For all analyses, the level of significance was fixed at $p<0.05$. 


\section{Results}

The final analysis included 186 RA patients $(68.8 \%$ female), with a mean (SD) age of 55.88 (14.36) years, and a mean (SD) disease duration of 5.31 (4.18) years. The $95.1 \%$ was right-hand dominating. At least one csDMARD or bDMARD was taken by all the patients. Fiftyfive $(29.5 \%)$ patients were receveing a bDMARD, respectively $15(27.3 \%)$ adalimumab, $14(25.4 \%)$ etanercept, $11(20.0 \%)$ golimumab, 9 (16.4\%) abatacept, 4 (7.2\%) tocilizumab, and 2 (3.6\%) infliximab. Thirty-two patients (17.2\%) were on oral corticosteroids, with a mean prednisone or equivalent dose of $4.9 \mathrm{mg} /$ day (range 2.5-20), while $113(60.8 \%)$ were given no-steroidal anti-inflammatory drugs on demand.

The mean values of the AUC-FeT, peak grip force, and time to reach the plateau of the curve were respectively $426.15(150.90) \mathrm{Kg} / 30 \mathrm{~s}, 17.51$ (6.03) $\mathrm{Kg}$, and 13.17 (6.32) s. Males patients have a higher AUC-FeT than females [472.46 (131.89) Kg/30 s vs 406.63 (154.90) $\mathrm{Kg} / 30 \mathrm{~s} ; p=0.014)$. Table 1 summarizes the clinical and demographic variables of the study cohort.

Figure 1 shows the estimation of central tendency and score distribution for the AUC-FeT scores, which resulted normal distributed.

Analyzing the relationships between the variables, Table 2 shows the correlations between handgrip strength findings and the other variables. The AUC-FeT was significantly correlated with age, SDAI, and US-CLARA (all with $p<0.0001)$, as well as with BMI $(p=0.0001)$. No correlations emerged with radiographic damage (SENS) and with comorbidities (mRDCI).

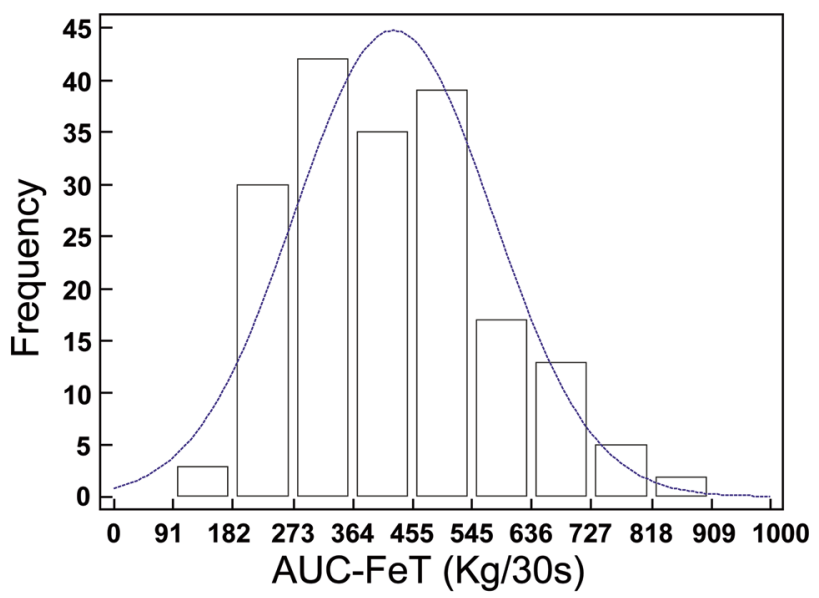

Fig. 1 Distribution of the area under the force-time curve (AUCFeT) scores

Categorizing patients according to SDAI categories, patients in HDA showed a significantly reduced AUC-FeT compared to patients in REM (ANOVA, F-ratio $=35.14$, $\mathrm{p}<0.001$ ) (Fig. 2). The AUC-ROC analyses revealed that the best performance in identifying RA patients in $\mathrm{MDA}+\mathrm{HDA}$ vs REM + LDA was provided by AUC-FeT [AUC-ROC $=0.810(95 \%$ CI 0.746-0.864)], while peak grip force and time to reach the curve plateau were not statistically significant (Fig. 3).

Finally, the multivariate analysis reveale that the independent variables significantly associated with AUC-FeT were age $(\mathrm{p}=0.0006)$, BMI $(\mathrm{p}=0.012)$, sex $(\mathrm{p}=0.004)$, SDAI $(\mathrm{p}=0.047)$, and US-CLARA $(\mathrm{p}=0.023)($ Table 3$)$.
Table 1 Demographic and clinical characteristics of the patients

\begin{tabular}{lrrrc}
\hline & Mean & Median & SD & \multicolumn{1}{c}{ IQR } \\
\hline Age (years) & 55.81 & 55.00 & 14.36 & $44.00-68.00$ \\
Disease duration (years) & 5.31 & 4.00 & 4.18 & $2.00-8.00$ \\
Educational level (years) & 11.66 & 13.00 & 3.91 & $8.00-13.00$ \\
BMI (Kg/m ${ }^{2}$ ) & 26.96 & 26.60 & 2.55 & $25.50-27.90$ \\
mRDCI (score 0-12) & 1.90 & 2.00 & 1.67 & $0.00-3.00$ \\
SDAI (score 0-86) & 22.02 & 18.40 & 14.86 & $10.20-33.44$ \\
US-CLARA (score 0-10) & 4.34 & 4.16 & 2.52 & $2.33-6.18$ \\
SENS (score 0-86) & 19.92 & 14.00 & 14.79 & $10.00-23.00$ \\
Handgrip strength AUC-FeT (Kg/30 s) & 426.15 & 418.08 & 150.90 & $303.25-515.36$ \\
Handgrip strength peak grip force (Kg) & 17.51 & 17.32 & 6.03 & $12.52-21.67$ \\
Time to reach maximum plateau of the curve (s) & 13.17 & 12.50 & 6.32 & $7.50-17.50$ \\
RF positivity, $\mathrm{n}(\%)$ & $141(75.80)$ & & & \\
ACPA positivity, n (\%) & $120(64.51)$ & & & \\
\hline
\end{tabular}

$S D$ standard deviation, $I Q R$ interquartile range, BMIbody mass index, $m R D C I$ modified Rheumatic Disease Comorbidity Index, SDAISimplified Disease Activity Index, US-CLARA Ultrasound-Clinical Arthritis Activity, SENS Simple Erosion Narrowing Score, $A U C$ - $F e T$ area under the force-time curve, $R F$ Rheumatoid Factor, $A C P A$ anti-citrullinated protein antibodies 


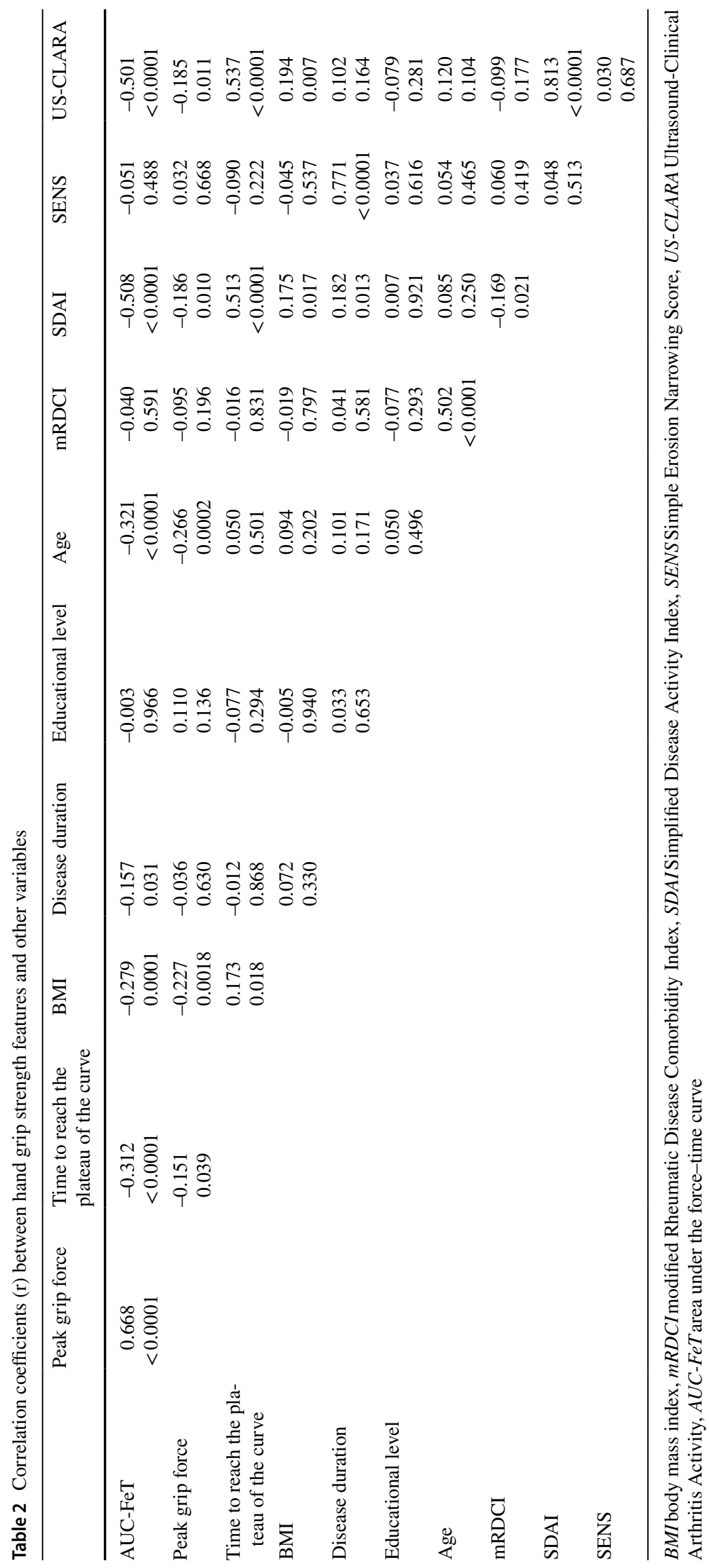




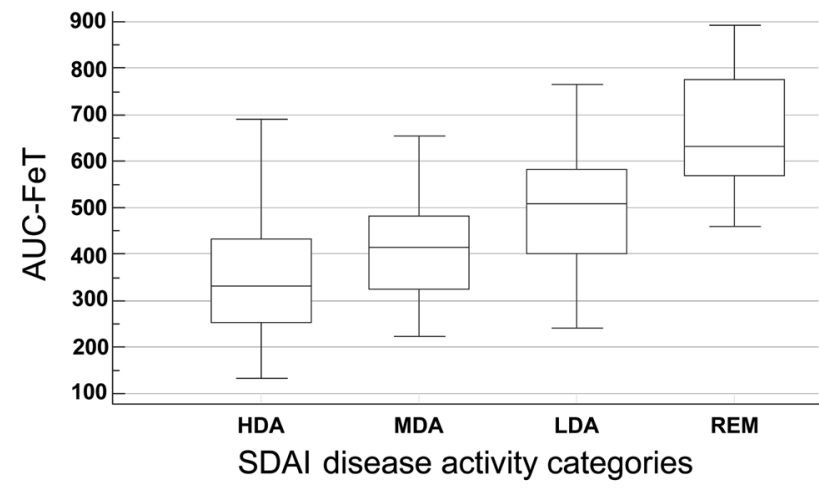

Fig. 2 Box-Whisker plots showing the relationship between the AUC-FeT scores and the disease activity states evaluated with SDAI (one-way analysis of variance: ANOVA, F-ratio $=35.14, \mathrm{p}<0.001$ ). The horizontal line in each box represents the median, and the box height represents the interquartile range

\section{Discussion}

HGs is one of the potential measures to capture muscular strength and is a simple and inexpensive risk-stratifying approach, having a prognostic value respect to all-causes mortality [24].

The objective of this study was to study the association between HGs features, estimated with an innovative cylindrical-shaped grip device, and demographic, anthropometric and clinical variables clinical variables in RA patients.

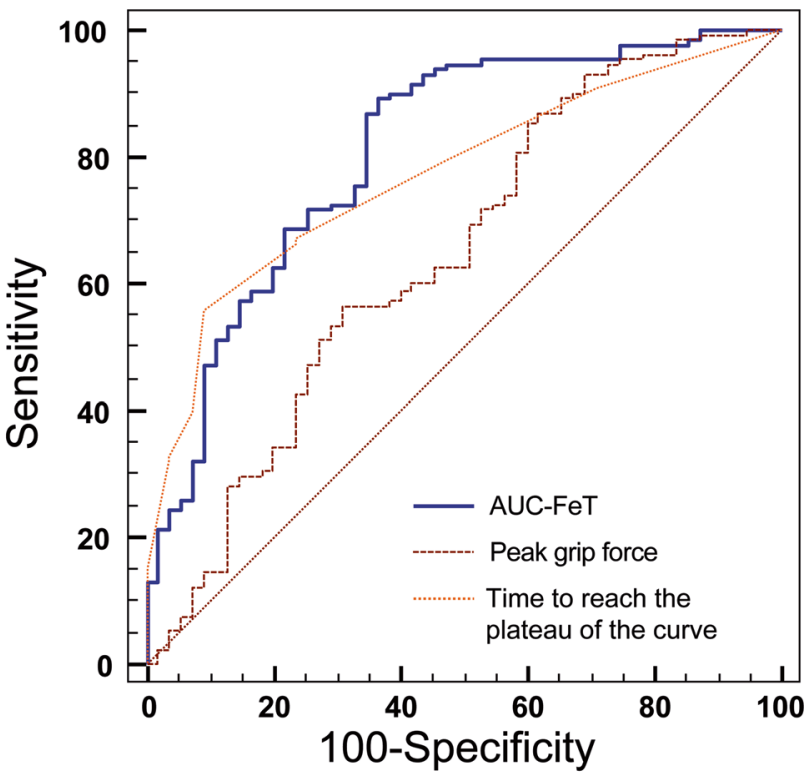

Fig. 3 Receiver operating characteristic curve plots comparing the discriminative power of each of the three handgrip strength measures in the identification of patients with moderate/high disease activity
Table 3 Variables associated with area under the force-time curve (AUC-FeT) in multivariate analysis

\begin{tabular}{lrrrl}
\hline Independent variables & Coefficient & Standard error & $\mathrm{t}$ & $\mathrm{p}$ \\
\hline (Constant) & 997.2249 & & & \\
Age & -2.5672 & 0.7370 & -3.484 & 0.0006 \\
Sex & -56.5544 & 19.7055 & -2.870 & 0.0046 \\
Education & -1.3249 & 2.3295 & -0.569 & 0.5703 \\
Disease duration & -2.5410 & 3.4591 & -0.735 & 0.4636 \\
BMI & -9.2652 & 3.6567 & -2.534 & 0.0122 \\
mRDCI & 2.4787 & 6.3206 & 0.392 & 0.6954 \\
SDAI & -2.4630 & 1.0631 & -2.317 & 0.0217 \\
US-CLARA & -14.1084 & 6.1664 & -2.288 & 0.0233 \\
SENS & 0.02848 & 0.9633 & 0.0296 & 0.9764 \\
\hline
\end{tabular}

$B M I$ body mass index, $m R D C I$ modified Rheumatic Disease Comorbidity Index, SDAISimplified Disease Activity Index, USCLARA Ultrasound-Clinical Arthritis Activity, SENSSimple Erosion Narrowing Score

In previous studies, HGs has been found to be related with disease activity scores and disability [6]. The results of our study confirm previous findings, namely that reduced HGs correlates with more severe disease activity, but also with parameters such as age and BMI.

The main innovation introduced in this study is the way in which HGs was assessed. Typically, a HGs test simply assesses only the maximum grip strength. Conventional dynamometers such as the Jamar or Takei assess only unidirectional isometric force. In the presence of potentially very complex clinical manifestations such as rheumatoid hand, a unidirectional assessment may not fully reflect the strength and skill required to handle a cylindrical object. It has been demonstrated that the force required to handle a cylindrical object is greater than that used on traditional dynamometers, just as a cylindrical instrument more reliably assesses the force applied to the distal portion of the fingers [25]. Moreover, the cylindrical-shaped grip device used in this study, allowed other misurations such as the speed with which strength is generated, the ability to sustain strength, and the variability of handgrip strength. These parameters are overlooked when measuring only the peak force. The importance of using a cylindrical-shaped grip device has already been demonstrated by our research group in the context of fibromyalgia [26].

Beyond the tool used, an immediate difference emerged between men and women. Males patients have higher mean HGs than females, just as they do in the general population [24]. The impact of sex on muscular mass, maximum strength, and consequently maximal physiological reserve differs significantly [27]. Maximum HGs in healthy women has been observed to be $52-80 \%$ reduced of those in men [28]. The AUC-FeT values of HGs in this investigation were $25 \%$ lower in women than in men. As a result, 
women with RA may be more susceptible to functional deficits because to their weaker muscles.

The progressive loss of muscle mass and strength is one the most ineluctable anatomical alteration that happens with age [29-32], and our study confirmed that age is the strongest predictor of a reduced AUC-FeT.

Obesity was found to be inversely related with physical activity in previous cross-sectional studies, among a variety of genetic, physiological, behavioral, and environmental factors influencing physical activity in RA [33]. Researchers found that baseline BMI strongly predicted later self-reported physical activity patterns in Danish adult populations in two earlier studies [34, 35]. A more recent study showed long-term relationships between many adiposity indicators and moderate-to-vigorous physical activity [36]. Inflammation in RA promotes the loss of metabolically active tissue, and fat mass tends to rise. This syndrome, also known as "rheumatoid cachexia" or "sarcopenic obesity," can worsen the disease by causing lethargy, weakness, and decreased functional status [37].

Regarding disease activity, the HGs variables were strongly associated to the composite and multimodal disease activity indices. HGs, pinch measurements, and clinical and laboratory activity indices were all significantly associated to hand impairment $[6,38,39]$. According to our findings, incorporating US-imaging into a multimodal disease activity measure could improve the accuracy of such systems and have major consequences for patient classification [14].

Controversial is the relationship between HGs and radiological damage, and the findings of studies examining the direct link between radiographic deterioration and functional impairment remain equivocal. In our analysis, the radiological damage did not correlate with HGs, which is consistent with previous findings [40, 41]. Another study demonstrated that articular damage is inversely related to grip strength as a handicap marker in RA [42].

One of our study's major flaws is the cross-sectional design, thus any possible prognostic importance of HGs in RA patients has yet to be discovered. Furthermore, the research did not allowed to verify if the HGs responds to physical and/or medical treatment. The sensitivity of the HGs test in detecting changes in symptomatology following a therapeutic intervention should be evaluated in future investigations. Another limitation may be represented by the fact that HGs was evaluated only through the cylindrical-shaped device, without performing a comparison of the innovative device with traditional dynamometers. Finally, it can be criticized that the scoring of radiological damage has been performed through the SENS, a simplified method of a more articulated scoring system such as the SHS. However, SENS has proven to be a comparable tool to SHS, reliable and timesaving $[15,43]$.
In conclusion, our findings revealed that HGs was predicted by demographic (gender and age), anthropometric (BMI), and disease activity indices (SDAI and US-CLARA) in RA patients. Radiological damage was not a predictor of a reduced HGs. Assessing HGs as a separate variable, using a cylindrical-shaped device, could aid clinicians in better understanding how RA impairs function.

Authors' contributions FS was the primary researcher, was responsible for coordinating and managing the study on a day-to-day basis, for data collection, data analysis and input into writing the manuscript. MDC provided support for the study, was involved in designing the study. MC provided clinical support and was involved in designing the study. SF and LC provided clinical support and contributed to revising the manuscript. All authors have read and approved the final manuscript.

Funding Open access funding provided by Università Politecnica delle Marche within the CRUI-CARE Agreement.

\section{Declarations}

Ethics approval All procedures performed in this study were approved by our institutional ethic review board (Comitato Etico Unico Regionale, numbee 20150458 AS) and were in accordance with the 1964 Helsinki Declaration and its later amendments or comparable ethical standards.

Consent to partecipate Written informed consent was obtained from all individual participants included in the study.

Consent for publication Not applicable.

Conflicts of interest/Competing interests All the authors declare that they have nor received any financial support or other benefits from commercial sources for the work reported in this manuscript, or any other financial interests that could create a potential conflict of interest or the appearance of a conflict of interest with regard to the work.

Open Access This article is licensed under a Creative Commons Attribution 4.0 International License, which permits use, sharing, adaptation, distribution and reproduction in any medium or format, as long as you give appropriate credit to the original author(s) and the source, provide a link to the Creative Commons licence, and indicate if changes were made. The images or other third party material in this article are included in the article's Creative Commons licence, unless indicated otherwise in a credit line to the material. If material is not included in the article's Creative Commons licence and your intended use is not permitted by statutory regulation or exceeds the permitted use, you will need to obtain permission directly from the copyright holder. To view a copy of this licence, visit http://creativecommons.org/licenses/by/4.0/.

\section{References}

1. Salaffi F, De Angelis R, Grassi W; MArche Pain Prevalence; INvestigation Group (MAPPING) study (2005) Prevalence of musculoskeletal conditions in an Italian population sample: results of a regional community-based study. I. The MAPPING study. Clin Exp Rheumatol 23:819-828. 
2. Fraser A, Vallow J, Preston A, Cooper RG (1999) Predicting 'normal' grip strength for rheumatoid arthritis patients. Rheumatology (Oxford) 38:521-528.

3. Salaffi F, Cimmino MA, Leardini G, Gasparini S, Grassi W (2009) Disease activity assessment of rheumatoid arthritis in daily practice: validity, internal consistency, reliability and congruency of the Disease Activity Score including 28 joints (DAS28) compared with the Clinical Disease Activity Index (CDAI). Clin Exp Rheumatol 27:552-559.

4. Eberhardt K, Sandqvist G, Geborek P (2008) Hand function tests are important and sensitive tools for assessment of treatment response in patients with rheumatoid arthritis. Scand J Rheumatol 37:109-112.

5. Escalante A, Haas RW, del Rincón I (2006) Measurement of global functional performance in patients with rheumatoid arthritis using rheumatology function tests. Arthritis Res Ther 6:R315-R325.

6. Bodur H, Yilmaz O, Keskin D (2006) Hand disability and related variables in patients with rheumatoid arthritis. Rheumatol Int 26:541-544.

7. Flipon E, Brazier M, Clavel G et al (2009) Is it possible to identify early predictors of the future cost of chronic arthritis? The VErA project. Fundam Clin Pharmacol 23:105-113.

8. Filippucci E, Cipolletta E, Mashadi Mirza R et al (2019) Ultrasound imaging in rheumatoid arthritis. Radiol Med 124:1087-1100.

9. Colebatch AN, Edwards CJ, Ostergaard M et al (2013) EULAR recommendations for the use of imaging of the joints in the clinical management of rheumatoid arthritis. Ann Rheum Dis 72:804-814.

10. Salaffi F, Filippucci E, Carotti M et al (2008) Inter-observer agreement of standard joint counts in early rheumatoid arthritis: a comparison with grey scale ultrasonography--a preliminary study. Rheumatology (Oxford) 47:54-58.

11. Carotti M, Salaffi F, Morbiducci J et al (2012) Colour Doppler ultrasonography evaluation of vascularization in the wrist and finger joints in rheumatoid arthritis patients and healthy subjects. Eur J Radiol 2012:1834-1838.

12. Damjanov N, Radunovic G, Prodanovic S et al (2012) Construct validity and reliability of ultrasound disease activity score in assessing joint inflammation in RA: comparison with DAS-28. Rheumatology (Oxford) 51:120-128.

13. Mandl P, Balint PV, Brault $Y$ et al (2013) Clinical and ultrasoundbased composite disease activity indices in rheumatoid arthritis: results from a multicenter, randomized study. Arthritis Care Res (Hoboken) 65:879-887.

14. Salaffi F, Di Carlo M, Iannone F et al (2018) The UltraSoundCLinical ARthritis Activity (US-CLARA) index: Properties of a new composite disease activity index for rheumatoid arthritis. Semin Arthritis Rheum 47:619-629.

15. van der Heijde D, Dankert T, Nieman F, Rau R, Boers M (1999) Reliability and sensitivity to change of a simplification of the Sharp/ van der Heijde radiological assessment in rheumatoid arthritis. Rheumatology (Oxford) 38:941-947.

16. Aletaha D, Neogi T, Silman AJ et al (2010) 2010 rheumatoid arthritis classification criteria: an American College of Rheumatology/ European League Against Rheumatism collaborative initiative. Arthritis Rheum 62:2569-2581.

17. Spaetgens B, Wijnands JM, van Durme C, Boonen A (2015) Content and construct validity of the Rheumatic Diseases Comorbidity Index in patients with gout. Rheumatology (Oxford) 54:1659-1663.

18. Iannone F, Salaffi F, Fornaro M et al (2018) Influence of baseline modified Rheumatic Disease Comorbidity Index (mRDCI) on drug survival and effectiveness of biological treatment in patients affected with Rheumatoid arthritis, Spondyloarthritis and Psoriatic arthritis in real-world settings. Eur J Clin Invest 48:e13013.

19. Haidar SG, Kumar D, Bassi RS, Deshmukh SC (2004) Average versus maximum grip strength: which is more consistent? J Hand Surg Br 29:82-84.
20. Fess EE (1992) Grip strength. In: American Society of Hand Therapists, ed. Clinical assessment recommendations. 2nd ed. Garner, NC: American Society of Hand Therapists, pp 41-45.

21. Aletaha D, Nell VP, Stamm T et al (2005) Acute phase reactants add little to composite disease activity indices for rheumatoid arthritis: validation of a clinical activity score. Arthritis Res Ther 7:R796-R806.

22. Salaffi F, Bazzichi L, Stancati A et al (2005) Development of a functional disability measurement tool to assess early arthritis: the Recent-Onset Arthritis Disability (ROAD) questionnaire. Clin Exp Rheumatol 23:628-636.

23. Salaffi F, Migliore A, Scarpellini M et al (2010) Psychometric properties of an index of three patient reported outcome (PRO) measures, termed the CLinical ARthritis Activity (PRO-CLARA) in patients with rheumatoid arthritis. The NEW INDICES study. Clin Exp Rheumatol 28:186-200.

24. Leong DP, Teo KK, Rangarajan S et al (2016) Reference ranges of handgrip strength from 125,462 healthy adults in 21 countries: a prospective urban rural epidemiologic (PURE) study. J Cachexia Sarcopenia Muscle 7:535-546.

25. McDowell TW, Wimer BM, Welcome DE, Warren C, Dong RG (2012) Effects of handle size and shape on measured grip strength. Int J Ind Ergon 42:199-205.

26. Salaffi F, Farah S, Di Carlo M (2020) Force-time curve features of handgrip strength in fibromyalgia syndrome. Sci Rep 10:3372.

27. Pedersen AN, Ovesen L, Schroll M, Avlund K, Era P (2002) Body composition of 80-years old men and women and its relation to muscle strength, physical activity and functional ability. J Nutr Health Aging 6:413-420.

28. Sinaki M, Nwaogwugwu NC, Phillips BE, Mokri MP (2001) Effect of gender, age, and anthropometry on axial and appendicular muscle strength. Am J Phys Med Rehabil 80:330-338.

29. Doherty T (2003) Aging and sarcopenia. J Appl Physiol 95:1717-1727.

30. Morley JE, Baumgartner RN, Roubenoff R, Mayer J, Nair KS (2001) Sarcopenia. J Lab Clin Med 137:231-243.

31. Roubenoff R (2003) Sarcopenia: effects on body composition and function. J Gerontol A Biol Sci Med Sci 58:1012-1017.

32. Schrager MA, Metter EJ, Simonsick E et al (2007) Sarcopenic obesity and inflammation in the InCHIANTI study. J Appl Physiol 102:919-925.

33. Besson H, Ekelund U, Luan J et al (2009) A cross-sectional analysis of physical activity and obesity indicators in European participants of the EPIC-PANACEA study. Int J Obesity 33:497-506.

34. Petersen L, Schnohr P, Sorensen TIA (2004) Longitudinal study of the long-term relation between physical activity and obesity in adults. Int J Obesity 28:105-112.

35. Bak H, Petersen L, Sorensen TIA (2004) Physical activity in relation to development and maintenance of obesity in men with and without juvenile onset obesity. Int J Obesity 28:99-104.

36. Golubic R, Wijndaele K, Sharp SJ et al (2015) Physical activity, sedentary time and gain in overall and central body fat: 7-year follow-up of the ProActive trial cohort. Int J Obesity 39:142-148.

37. Rall LC, Roubenoff R (2004) Rheumatoid cachexia: metabolic abnormalities, mechanisms and interventions. Rheumatology (Oxford) 43:1219-1223.

38. Palamar D, Er G, Terlemez R, Ustun I, Can G, Saridogan M (2017) Disease activity, handgrip strengths, and hand dexterity in patients with rheumatoid arthritis. Clin Rheumatol 36:2201-2208.

39. Nampei A, Shi K, Hirao M, Murase T, Yoshikawa H, Hashimoto J (2011) Association of pinch strength with hand dysfunction, finger deformities and contact points in patients with rheumatoid arthritis. Clin Exp Rheumatol 29:1061.

40. Salaffi F, Ferraccioli GF, Troise Rioda W, Marini M, Cervini C (1992) No relationship between anatomic damage and disability in 
rheumatoid arthritis (RA). A retrospective assessment. Clin Rheumatol 11:582-583.

41. Birtane M, Kabayel DD, Uzunca K, Unlu E, Tastekin N (2008) The relation of hand functions with radiological damage and disease activity in rheumatoid arthritis. Rheumatol Int 28:407-412.

42. Dedeoglu M, Gafuroglu Ü, Yilmaz Ö, Bodur H (2013) The relationship between hand grip and pinch strengths and disease activity, articular damage, pain, and disability in patients with rheumatoid arthritis. Arch Rheumatol 28:69-77.
43. Guillemin F, Billot L, Boini S, Gerard N, Ødegaard S, Kvien TK (2005) Reproducibility and sensitivity to change of 5 methods for scoring hand radiographic damage in patients with rheumatoid arthritis. J Rheumatol 32:778-786.

Publisher's Note Springer Nature remains neutral with regard to jurisdictional claims in published maps and institutional affiliations.

\section{Authors and Affiliations}

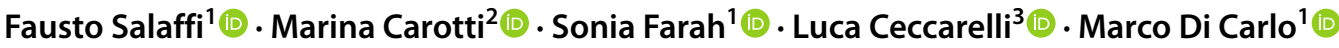

Fausto Salaffi

fausto.salaffi@gmail.com

Marina Carotti

marina.carotti@gmail.com

Sonia Farah

sonia.farah91@gmail.com

Luca Ceccarelli

luca.ceccarelli28@gmail.com
1 Rheumatology Clinic, Ospedale "Carlo Urbani”, Università Politecnica delle Marche, Jesi (Ancona), Italy

2 Department of Radiology, Ospedali Riuniti, Università Politecnica delle Marche, Ancona, Italy

3 U.O. di Radiologia - Ospedale degli Infermi, Azienda AUSL della Romagna, Faenza, Italy 